\title{
Model Predictive Control Design for Nonlinear Process Control Reactor Case Study: CSTR (Continuous Stirred Tank Reactor)
}

\author{
Mrs. M. Shyamalagowri, M.E., (Ph.D) ${ }^{1 *}$, Dr. R. Rajeswari., M.E., Ph.D ${ }^{2}$ \\ ${ }^{*}$ Corresponding Author, Research Scholar, Assistant Professor / Selection Grade - II,Erode Sengunthar \\ Engineering College, Thudupathi, Erode \\ ${ }^{2}$ Asst Prof (Senior) / EEE, Government College of Technology, Coimbatore
}

\begin{abstract}
Continuous Stirred Tank Reactor (CSTR) is an essential focus in process control and offering a diverse range of researches in the area of chemical and control engineering. To ensure the successful operation of a continuous stirred tank reactor (CSTR), it is necessary to understand their dynamic characteristics, which ultimately enable effective control systems design. The problem of controlling the nonlinearity of CSTR is considered as a challenging issue especially for a control engineer corresponding to its non linear dynamics. Most of the traditional controllers are restricted just for linear time invariant system application. But in real world, the non linear characteristics of system and their function parameter changes due to wear and tear, that's why these changes can't be neglected. A simulation on mathematical model has several advantages over the experiment on a real model or system, which is used for steady state analysis and dynamic state analysis. Model predictive control (MPC) has been the most successful advanced control technique applied in the process industries, nowadays recognized as a standard methodology for the control of industrial and process systems. In this approach, a process model is used to predict the effect of a finite number of future moves on the controlled variable. From the transient responses of the controlled variable to the changes in manipulated variable, a dynamic network has been developed for prediction of one time step a forward in future process output with good accuracy. MPC refers to a class of computer controlled algorithms that utilize and explicit process model to predict the future response of the plant. At each control interval, an MPC algorithm attempts to optimize future plant behavior by computing a sequence of future manipulated variable adjustments. The first input in the optimal sequence is then sent into the plant and entire calculation is repeated at subsequent control intervals. The proposed MPC structure is to have a control system that will be able to achieve improvement in the level of conversion and to be able to track set point change and reject load disturbance. The simulation results confirm that the MPC is one of the best possibilities for successful control of nonlinear systems.
\end{abstract}

Key Words: Continuous Stirred Tank Reactor (CSTR), Model Predictive Controller (MPC), Nonlinearity, Multi Input Multi Output (MIMO)

\section{Introduction}

Chemical reactors are the most influential and therefore the important units that a chemical engineer will encounter. The aim is to introduce some basic concepts of chemical reaction system modeling and develop simulation models for CSTR's in which the non-linear and linear system descriptions are derived. CSTR is one of the major processing units in chemical engineering such a problem remains too complex to be solved by the known techniques. Chemical process control requires intelligent monitoring due to the dynamic nature of the chemical reactions and non- linear functional relationships between the input and output variables are involved, therefore, model predictive control can provide good optimal solutions for many applications. With the advent of high-speed computer system, there is more increase interest in the study of non-linear system. The need for this interest is born out of the fact that most industrial process is inherently non-linear and reasons, which include, higher quality specification, improvement in productivity, tighter regulations on environment, and demand for operating safety and high quest for economic profitability have called for to operate system over the boundary of a wide range of operating conditions and often near the boundary of a admissible region. In modern processing plants the MPC Controller is part of a multi level hierarchy of control functions. Model design and simulation are done in MATLAB SIMULINK. 
II. Cstr System Description

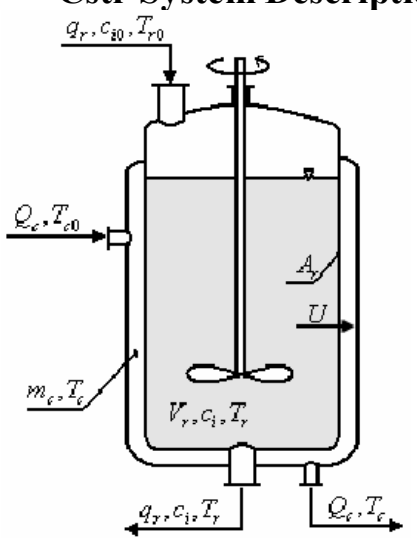

Fig No.1 Schematic Diagram of the CSTR

The examined system is represented by Continuous stirred Tank Reactor (CSTR) which is widely used in the process industries, which is the nonlinearity identification process. The Schematic diagram of CSTR is given in the figure. It has a jacket surrounding the reactor also has feed and exit streams. It is assumed to be perfectly mixed at lower temperature than the rector. Energy passes through the reactor walls into jacket removing the heat generated by reaction. The control objective is to keep the temperature of the reacting mixture $\mathrm{T}$, constant at desired value. The only manipulated variable is the coolant flow rate. It consists of a CSTR with a cooling jacket carrying out the Vander Vusse reaction scheme described by the following reactions:

$$
\begin{gathered}
A \stackrel{k 1}{\rightarrow} B \stackrel{k 2}{\rightarrow} C \\
2 A \stackrel{k 3}{\rightarrow} D .
\end{gathered}
$$

Here B is the desired product,

$\mathrm{C}$ and $\mathrm{D}$ are the undesired byproducts

$\mathrm{k}_{1}, \mathrm{k}_{2}$ and $\mathrm{k}_{3}$ are the reaction rate constants.

In this reactor, a product $\mathrm{A}$ is to be converted to the desired product $\mathrm{B}$, in an exothermic CSTR, but the product $\mathrm{B}$ is degraded to product $\mathrm{C}$. In addition to this consecutive reaction, a high order parallel reaction occurs and $\mathrm{A}$ is converted to by product $\mathrm{D}$. The mathematical model of this reactor is described by the set of four Ordinary Differential Equations (ODE) which come from material and heat balances inside the reactor.

$$
\begin{aligned}
& \frac{d C_{A}}{d t}=\frac{q_{r}}{V_{r}}\left(C_{A 0}-C_{A}\right)-k_{1} C_{A}-k_{3} C_{A}^{2} \\
& \frac{d C_{B}}{d t}=-\frac{q_{r}}{V_{r}} C_{B}+k_{1} C_{A}-k_{2} C_{B^{-}} \\
& \frac{d T_{r}}{d t}=\frac{q_{r}}{V_{r}}\left(T_{r 0}-T_{r}\right)-\frac{h_{r}}{\rho_{r} c_{p r}}+\frac{A_{r} U}{V_{r} \rho_{r} c_{p r}}\left(T_{c}-T_{r}\right) \\
& \frac{d T_{c}}{d t}=\frac{1}{m_{c} c_{p c}}\left(Q_{c}+A_{r} U\left(T_{r}-T_{c}\right)\right)
\end{aligned}
$$

Where $\mathrm{C}_{\mathrm{A}} \geq 0, \mathrm{C}_{\mathrm{B}} \geq 0$

In the set of equations $t$ is the time, $c$ are concentrations, $T$ represents temperatures, $c_{p}$ is used for specific heat capacities, $q$ represents volumetric flow rate, $Q_{c}$ is heat removal, $V$ are volumes, $\rho$ represents densities, $A_{r}$ is the heat exchange surface and $\mathrm{U}$ is the heat transfer coefficient. Indexes $(.)_{\mathrm{A}}$ and $(.)_{\mathrm{B}}$ belongs to compounds $\mathrm{A}$ and $\mathrm{B},(.)_{\mathrm{r}}$ denotes the reactant mixture, $(.)_{\mathrm{c}}$ cooling liquid and (.) are feed (inlet) values. The concentrations $\mathrm{C}_{\mathrm{A}}$ and $\mathrm{C}_{\mathrm{B}}$, reactor temperature $\mathrm{T}$ and the coolant temperature $\mathrm{T}_{\mathrm{c}}$ constitute the four states of the plant.

The model of the reactor belongs to the class of lumped parameter nonlinear systems. Nonlinearity can be found in reaction rates $\left(\mathrm{k}_{\mathrm{j}}\right)$ which are described via Arrhenius law:

$$
k_{j}\left(T_{r}\right)=k_{0 j} \cdot \exp \left(\frac{-E_{j}}{R T_{r}}\right), \text { for } j=1,2,3
$$


Where $\mathrm{k}_{0}$ represent pre-exponential factors and $\mathrm{E}$ are activation energies.

The reaction heat $\left(\mathrm{h}_{\mathrm{r}}\right)$ in the equation (2) is expressed as:

$$
h_{r}=h_{1} k_{1} c_{A}+h_{2} k_{2} c_{B}+h_{3} k_{3} c_{A}^{2}
$$

Where $h_{j}$ means reaction enthalpies.

Table 1: PARAMETERS OF THE REACTOR

\begin{tabular}{|l|l|l|}
\hline$k_{01}=2.145 \cdot 10^{10} \mathrm{~min}^{-1}$ & $k_{02}=2.145 \cdot 10^{10} \mathrm{~min}^{-1}$ & $k_{03}=1.5072 \cdot 10^{8} \mathrm{~min}^{-1} \mathrm{~mol}^{-1}$ \\
$E_{1} / R=9758.3 \mathrm{~K}$ & $E_{2} / R=9758.3 \mathrm{~K}$ & $E_{3} / R=8560 \mathrm{~K}$ \\
$h_{1}=-4200 \mathrm{~kJ} \cdot \mathrm{kmol}^{-1}$ & $h_{2}=11000 \mathrm{~kJ} \cdot \mathrm{kmol}^{-1}$ & $h_{3}=41850 \mathrm{~kJ} \cdot \mathrm{kmol}^{-1}$ \\
$V_{r}=0.01 \mathrm{~m}^{3}$ & $\rho_{r}=934.2 \mathrm{~kg} \cdot \mathrm{m}^{-3}$ & $c_{p r}=3.01 \mathrm{~kJ}^{-1} \mathrm{~kg}^{-1} \cdot \mathrm{K}^{-1}$ \\
$U=67.2 \mathrm{~kJ} \cdot \mathrm{min}^{-1} \mathrm{~m}^{-2} \mathrm{~K}^{-1}$ & $c_{p c}=2.0 \mathrm{~kJ} \cdot \mathrm{kg}^{-1} \cdot \mathrm{K}^{-1}$ & $A_{r}=0.215 \mathrm{~m}^{2}$ \\
$c_{A 0}=5.1 \mathrm{kmol} \cdot \mathrm{m}^{-3}$ & $T_{r 0}=387.05 \mathrm{~K}$ & $U=67.2 \mathrm{~kJ} \cdot \mathrm{min}^{-1} \mathrm{~m}^{-2} \mathrm{~K}^{-1}$ \\
$m_{c}=5 \mathrm{~kg}$ & $c_{B 0}=0 \mathrm{kmol} \cdot \mathrm{m}^{-3}$ & \\
\hline
\end{tabular}

This reaction describes the chemical conversion, under ideal conditions, of an inflow of substance A to a product B. For controlling the heat inside the reactor, a heat exchanger with a coolant flow is used. To simplify the problem the following assumptions are taken:

- The liquid in the reactor is ideally mixed.

- The density and the physical properties are constant.

- The liquid level $\mathrm{h}$ in the tank is constant, implying that the input and output flows is equal: $\mathrm{Q} 1=\mathrm{Q} 2$.

- The reaction is first order with a temperature relation according to the Arrhenius law.

- The shaft work can be neglected.

- The temperature increase of the coolant over the coil can be neglected.

It suffices to know that within the CSTR two chemicals are mixed and react to produce a product compound A at a concentration $C_{A}(t)$, with the temperature of the mixture being $T(t)$. The reaction is exothermic and producing heat which slows down the reaction. By introducing a coolant flow-rate $\mathrm{Q}_{c}(\mathrm{t})$, the temperature can be varied and hence the product concentration controlled. $\mathrm{C}_{\mathrm{A}}$ is the inlet feed concentration, $\mathrm{Q}$ is the process flow-rate, $\mathrm{T}$ and $\mathrm{T}_{\mathrm{C}}$ are the inlet feed and coolant temperatures, respectively, all of which are assumed constant at nominal values.

\section{Model Predictive Control}

Model Predictive Control is the only advanced control technique, which has been very successful in particular applications. Model predictive control (MPC) refers to a class of computer control algorithms that control the future behavior of a plant through the use of an explicit process model. At each control interval the MPC algorithm computes an open-loop sequence of manipulated variable adjustments in order to optimize future plant behavior. The Model Predictive Control problem is formulated as solving on-line a finite horizon open loop optimal control problem subject to system dynamics and constraints involving states and controls. Fig shows the basic principle of model predictive control. Based on measurements obtained at time $t$, the controller predicts the future dynamic behavior of the system over a prediction horizon $T$ and determines (over a control horizon) the input such that a predetermined open-loop performance object function is optimized. 


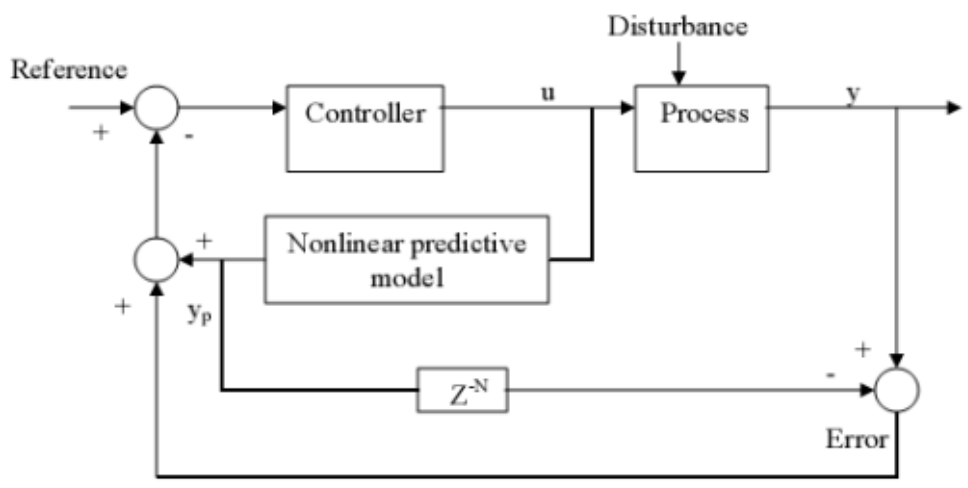

Fig. 2. Block diagram of the MPC system.

Model predictive control (MPC) is a very attractive concept for the development and tuning of nonlinear controllers in the presence of input, output or state constraint. The first input in the optimal sequence is injected into the plant, and the entire optimization is repeated at subsequent control intervals. MPC technology was originally developed for power plant and petroleum refinery applications, but can now be found in a wide variety of manufacturing environments including chemicals, food processing, automotive, aerospace, metallurgy and pulp and paper. The application of MPC controllers based on linear dynamic models cover a wide range of applications, and linear MPC theory can be considered quite mature. Nevertheless, many manufacturing processes are inherently nonlinear and there are cases where nonlinear effects are significant and can-not be ignored. These include at least two broad categories of applications:

1. Regulator control problems where the process is highly nonlinear and subject to large frequent disturbances $(\mathrm{pH}$ control, etc.).

2. Servo control problems where the operating points change frequently and span a wide range of nonlinear process dynamics (polymer manufacturing, ammonia synthesis, etc.).

Model based predictive control, MBPC, strategy has received particular attention in the areas of process control, is based on the use of a model for predicting the future behaviors of the system over a finite future horizon. The control signal to be applied to the plant at the current sampling time is obtained by solving a finite dimension optimization problem over the prediction horizon. "MPC is the family of controllers in which there is a direct use of an explicit and separately identifiable model"

The advantages of MPC compared with many other control techniques can be listed as follows:

- It can use step and impulse response data which can easily be obtained,

- It can handle input/output constraints directly,

- It gives satisfactory performance even with time delays and high nonlinearities,

- It can be used in multivariable format,

- It is robust in most cases,

- Implementation of the technique is simple,

- It can optimize over a trajectory,

- It can be used to control various processes, whether simple or complex ones.

The sum of squared difference between the predicted outputs and their set points over the future prediction horizon and the sum of squares of the control moves over the control horizon. The control of a process, quantitative formulation of the control objective is to minimize the cost

$\min J\left(N_{1}, N_{2}, U\right)=\lambda_{y} \sum_{i=N 1}^{N 2}\left[r(t+i)-y_{p}(t+i)\right]^{2}+\lambda_{U} \sum_{i=1}^{N U}[\Delta u(t+i-1)]^{2}$

Subject to the constraints

$\mathrm{N}_{\mathrm{U}} \leq \mathrm{N}_{1,2}$

$|\Delta \mathrm{u}(\mathrm{t})| \leq \Delta \mathrm{u}_{\max }$

$\mathrm{u}_{\min } \leq \mathrm{u}(\mathrm{t}) \leq \mathrm{u}_{\max }$

$\mathrm{y}_{\mathrm{pmin}} \leq \mathrm{y}_{\mathrm{p}} \leq \mathrm{y}_{\mathrm{pmax}}$ 
where $r$ is the reference, $y_{p}$ is the predicted output, $u$ is the control variable, $\lambda_{y}$ and $\lambda_{U}$ are the output and the input weighting parameter: $\mathrm{N}_{1}, \mathrm{~N}_{2}$ represents minimum and maximum prediction horizon and $\mathrm{N}_{U}$ is the controller moves horizon. Here $\mathrm{U}$ is the $\mathrm{N}_{\mathrm{U}}$ future inputs vector defined as, $U=\left[u(t), u(t+1), \ldots \ldots \ldots . . u\left(t+N_{U}-1\right)\right]^{T}$

\section{Simulation Results And Discussion}

In this paper presents the simulation result of model predictive controller design for nonlinear process control reactor - Continuous Stirred Tank Reactor without noise. In this reactor, a product A is to be converted to the desired product $\mathrm{B}$, in an exothermic CSTR, but the product B is degraded to product $\mathrm{C}$. In addition to this consecutive reaction, a high order parallel reaction occurs and $\mathrm{A}$ is converted to by product $\mathrm{D}$. in the reaction heat produced inside the reactor is a nonlinear process. The controller design for CSTR produces the optimum solution for the variation in parameters of the reactor. MPC algorithm proves that, it produces best controller design and solution for nonlinearities in CSTR. The simulation result presents the measured disturbances, measured variable and output/reference/noise waveforms.

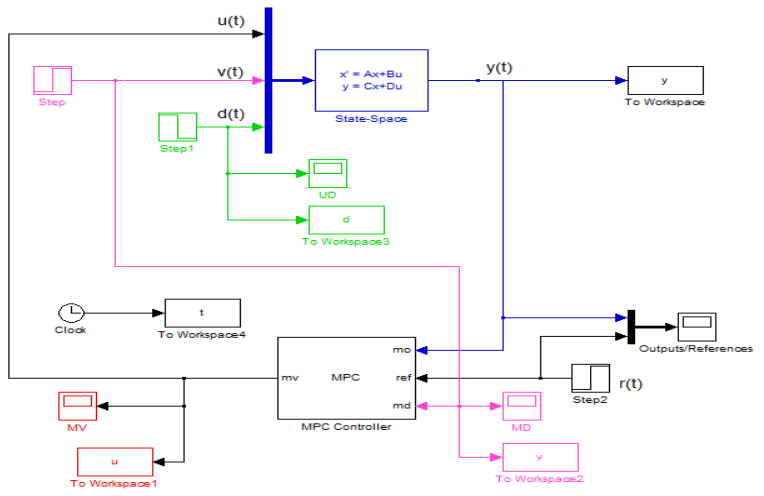

Fig No.3 Simulation diagram of Model Predictive Controller without noise

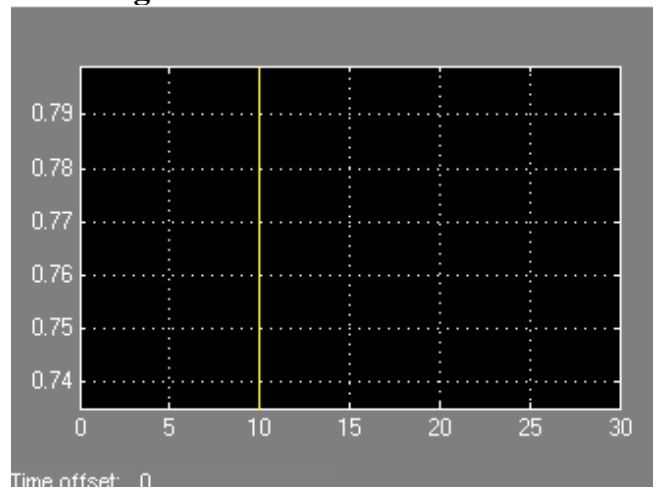

Fig No.4 Measured disturbance to the MPC model

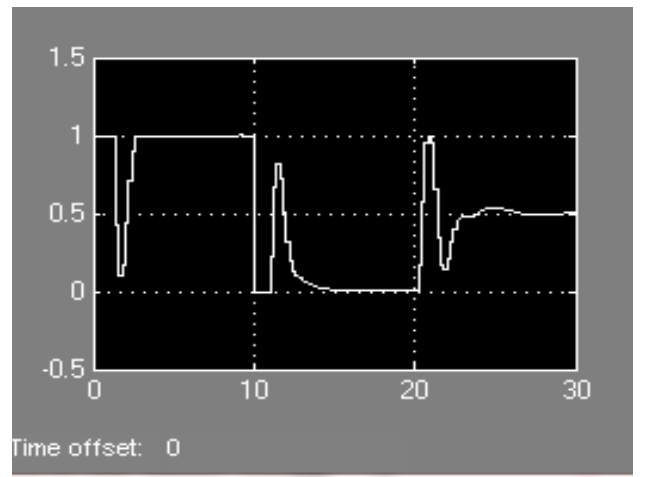

Fig No.5 Simulation Result - Measured variable 


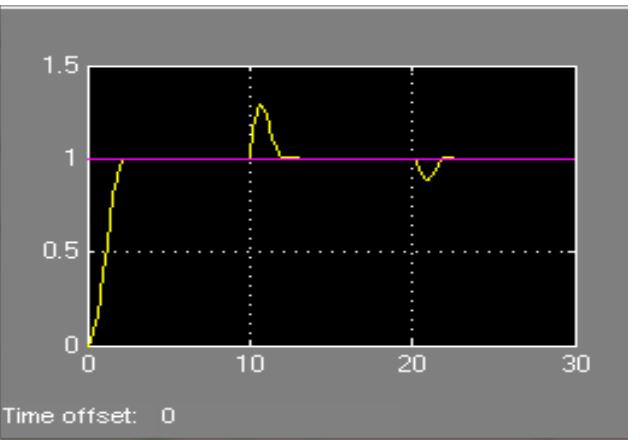

Fig No.6 Simulation Result - Output / Reference

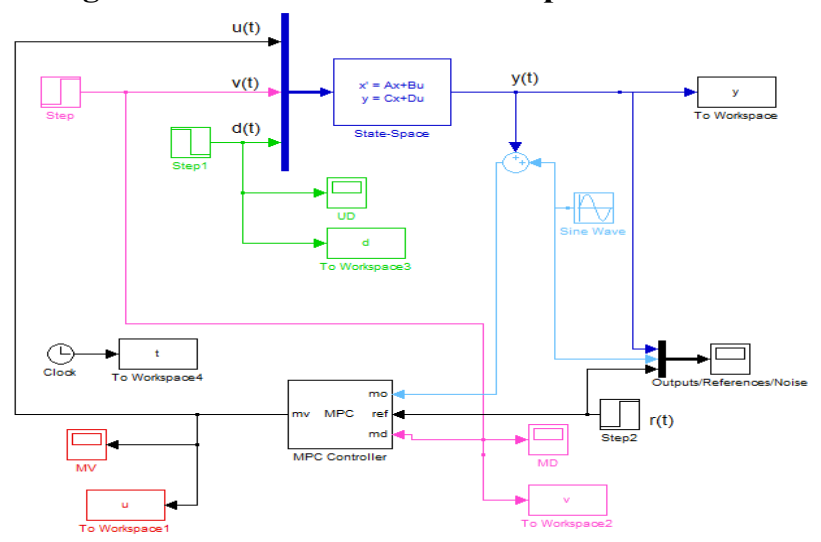

Fig No.7 Simulation diagram of Model Predictive Controller with noise

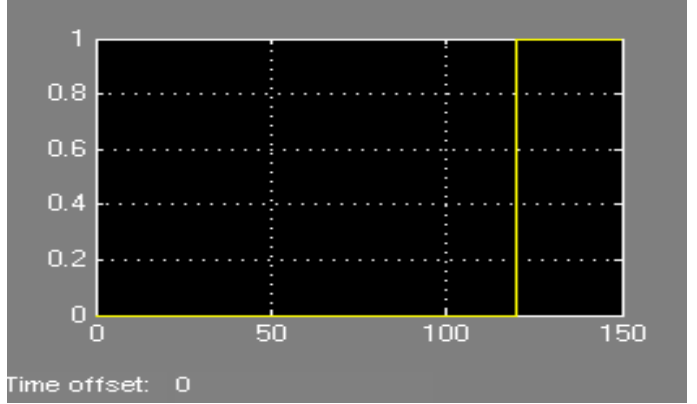

Fig No.8 Measured disturbance to the MPC model

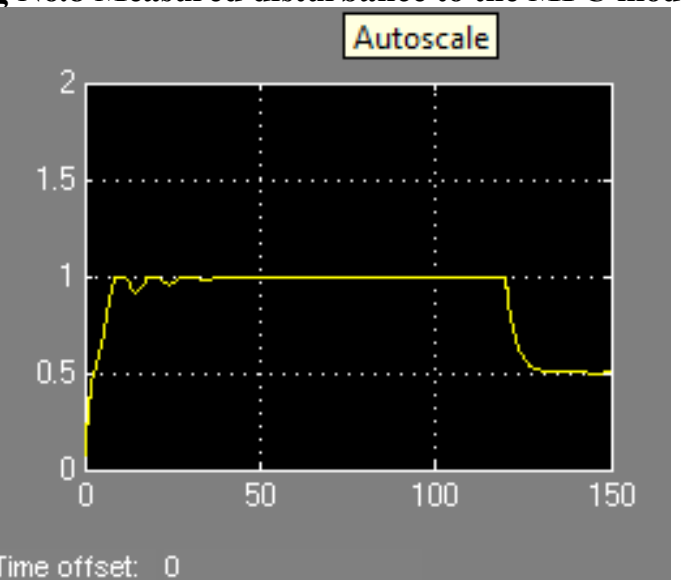

Fig No.9 Simulation Result - Measured variable 


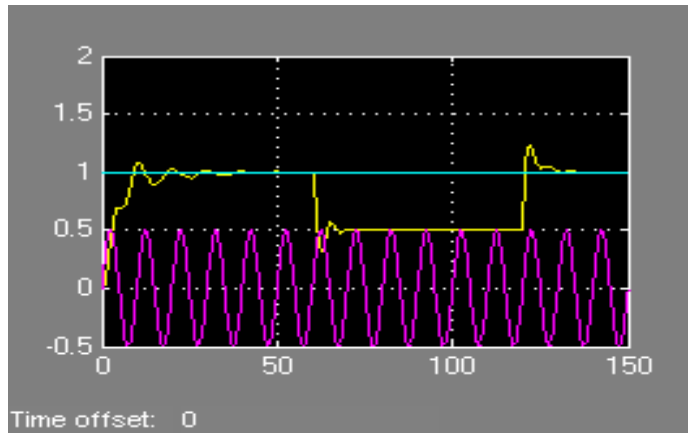

Fig No.10 Simulation Result - Output / Reference \& Noise

\section{Conclusion}

The CSTR process is identified as a strong non-linear system. The modeling of CSTR process is implemented with the help of first principle differential equation. The MPC Controller is implemented to track the servo response and regulatory response. The simulation results proven that the MPC control method is an easytuning and more effective way to enhance stability of time domain performance of the temperature control of CSTR process. The simulation results demonstrate the capability of the proposed identification strategy to effectively identify compact, accurate and transparent model for the CSTR process. It is shown that the proposed model predictive control design presents better improvement with faster response time for both servo and regulatory control objectives. Model Predictive Control (MPC) approaches have been recognized as the accepted standard to cope with some of the difficult control problems in process industry.

\section{References}

[1] V.R.Ravi, T.Thyagarajan, "Application of adaptive control technique to interacting Non Linear Systems", IEEE 2011, pp 386-392.

[2] Pan. Y., Wang Jun. Model predictive control of unknown nonlinear dynamical systems based on recurrent neural networks , IEEE Transactions on Industrial Electronics, vol. 59, no. 8, 2012, pp. 3089 to 3101.

[3] Rahideh. A., Shaheed. M. H. Constrained output feedback model predictive control for nonlinear systems, Control Engineering Practice, vol. 20, no. 4, pp. 431 to $443,2012$.

[4] Sukanya R.warier, Sivanandamvenkatesh "Design of controller based on MPC for a conical tank system”,(ICAESM-2012).

[5] Tuan. T. Q., Minh. Phan Xuan. Adaptive Fuzzy Model predictive control for non-minimum phase and uncertain dynamical nonlinear systems, Journal of Computers, vol. 7, no. 4, 2012, pp. 1014 to 1024.

[6] Qiao Ji-Hong, Wang Hong-Yan. Backstepping Control with Nonlinear Disturbance Observer for Tank Gun Control System, Control and Decision Conference (CCDC), 2011 Chinese, 2011, pp. 251 to 254.

[7] Hong Man, Cheng Shao. Nonlinear model predictive control based on LS-SVM Hammersteinwiener model, Journal of Computational Information Systems, vol. 8, no. 4, 2012, pp. 1373 to 1381.

\section{AUTHORS}

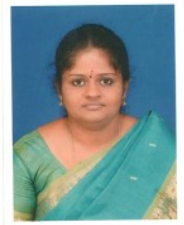

Mrs. M.Shyamalagowri received the B.E degree in Electrical and Electronics Engineering from KSR College of Technology, Thiruchengode in 2001 and M.E., Control Systems from PSG College of Technology, Coimbatore in 2006. She is currently working for Ph.D degree in the area of Control Systems at Anna University of Technology, Coimbatore. Her area of interest is Control Systems, Process Control. She is working as a Assistant Professor in EEE Department in the Erode Sengunthar Engineering College having the experience of 10 years 8 months.

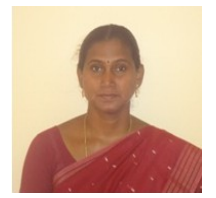

Dr. R.Rajeswari received the B.E.(EEE) Degree from TCE, MK University in the year 1995 and M.E.(Power System Engineering) from TCE, MK University in the year 1998. She has completed the Doctoral Degree from Anna University, Chennai in the area of Power System Protection in the year 2009. Her area of interest is Power System Engineering. Presently she is working as Asst Prof (Senior) / EEE at Government College of Technology, Coimbatore. 